\title{
Reduction of anterior dislocation of the shoulder
}

Sir

I refer to the letter by A. Banerjee (1990) regarding reduction of anterior dislocation of the shoulder without anaesthesia or analgesia. Proponents of this technique may have the knack of easily reducing some dislocated shoulders without pharmacological assistance, especially immediately after the traumatic event. However, such a procedure will cause unnecessary pain and anxiety in many patients. Moreover, if any undue force be applied by the operator in the face of a lack of any muscle relaxation, further damage to the glenoid labrum, articular capsule and cartilage may occur.

The gold standard has to be a general anaesthetic, combining 'hypnosis', analgesia and muscular relaxation, used definitively and not as a procedure to fall back upon. The regional technique of choice is probably interscalene block (Underhill et al., 1989). Both methods require expertise and time, and are not appropriate in an accident and emergency (A\&E) department setting. Kendall (1991) has outlined his preference for intravenous analgesia and Entonox. The practice in our department is similar but has a few additions.

Firstly, all adult patients receive intravenous metoclopramide $10 \mathrm{mg}$ as antiemetic prior to IV opiates.

Secondly, following initial radiography and transfer of the patient to a suite with resuscitation and monitoring facilities, all patients receive $40-50 \%$ oxygen by mask. Supplemental IV opiates may be required.

Thirdly, IV midazolam is given slowly to a maximum of $5 \mathrm{mg}$. This benzodiazepine is preferred because of its short duration of action and its tendency to produce amnesia for the reduction, as well as sedation with some direct muscle relaxation.

Finally, we use ECG and BP monitoring, and hypoxia is guarded against by pulse oximetry (Holburn et al., 1989). This requires the presence of a second doctor.

To date, we have not failed to secure reduction with this method. Treatment is delivered promptly. Patients find it highly acceptable and are able to go home on the same day.

The reduction of dislocated shoulders without any adjuncts harks back to the Victorian Age of surgery and has very little to commend it except speed and simplicity. In modern A\&E departments, doctors should make full use of pharmacotherapy and technology whenever they are able for the comfort and safety of all their patients. There are reasonable theoretical grounds for believing that such practice might prevent or reduce long term morbidity, such as recurrent dislocation.

\section{SEDGWICK}

Registrar in Accident \& Emergency Medicine,

Basingstoke District Hospital,

Basingstoke

\section{REFERENCES}

Banerjee A. (1990) Is anaesthesia necessary for reducing shoulder dislocation? Archives of Emergency Medicine 7, 240. 
Holburn C. J. \& Allen M. J. (1989) Pulse oximetry in the accident and emergency department. Archives of Emergency Medicine 6, 137.

Kendall I. G. (1991) Is anaesthesia necessary for reducing shoulder dislocations? Archives of Entergency Medicine 8, 288.

Underhill T. J., Wan A. \& Morrice M. (1989) Interscalene brachial plexus blocks in the management of shoulder dislocation. Archives of Emergency Medicine 6, 199.

\section{Portable $\mathrm{CO}_{2}$ detectors}

Sir

We read with interest the equipment review of the MiniCAP III $\mathrm{CO}_{2}$ Detector (Archives of Emergency Medicine, 1992, 9, 373-376). We have had considerable experience in the use of this and similar portable $\mathrm{CO}_{2}$ detectors in the pre-hospital care of patients and can confirm its value. Road traffic accidents, particularly those where the patient requires extrication from wreckage are frequently extremely noisy. It is not always possible to assess accurately correct positioning of an endotracheal tube using auscultation alone. It is our practice to use a portable $\mathrm{CO}_{2}$ monitor in concert with a pulse oximiter in these difficult field conditions.

We also agree that an alarm, which may indicate that an endotracheal tube has become misplaced or disconnected, is invaluable in the often fast and bumpy ride between the accident scene and hospital. The audible alarm on such devices enables the practitioner to concentrate on other life threatening injuries to the same, or perhaps other patients, secure in the knowledge that should the endotracheal tube become dislodged, or the patient deteriorate, then he will be warned immediately.

J. M. HOPPER \& K. M. PORTER

The Central Accident Resuscitation, and Emergency (CARE) Team, c/o Birmingham Accident Hospital, Bath Row,

Birmingham

\section{REFERENCE}

McLeod G. A. \& Inglis M. D. (1992; ; _ pe MiniCAP III CO $\mathrm{CO}_{2}$ Detector: assessment of a device to distinguish oesophageal from tracheal intuba...... . irchive of Entergency Medicine 9, 373-376.

The effect of the Cornwall and Isles of Scilly helicopter ambulance unit on the ambulance services ability to deliver acutely traumatized patients to hospital

Sir

There can be no doubt that in order to give helicopter ambulances a proper clinical audit there is a need for a prospective study. The paper by Rouse (1992) has no new messages, particularly as 14 out of the 17 cases involving the deployment of a 